\title{
Expected Sample Moments of Concomitants of Selected Order Statistics
}

\author{
Dirk V. Arnold and Hans-Georg Beyer \\ Department of Computer Science XI \\ University of Dortmund \\ 44221 Dortmund, Germany
}

\begin{abstract}
In this paper, the task of determining expected values of sample moments, where the sample members have been selected based on noisy information, is considered. Exact expressions for expected values of sums of products of concomitants of selected order statistics are derived. Then, using Edgeworth and Cornish-Fisher approximations, explicit results that depend on coefficients that can be determined numerically are obtained. While the results are exact only for normal populations, it is shown experimentally that including skewness and kurtosis in the calculations can yield greatly improved results for other distributions.
\end{abstract}

Keywords: Concomitants of order statistics, Gaussian noise, sample moments, Edgeworth approximation, Cornish-Fisher expansion

\section{Introduction}

Suppose that $\left(X_{1}, Y_{1}\right), \ldots,\left(X_{\lambda}, Y_{\lambda}\right)$ are $\lambda$ i.i.d. bivariate observations from a continuous population with c.d.f. $F(x, y)$ and with p.d.f. $f(x, y)$. Further, suppose that the points are ordered by their $X$ variates. The order statistics of $X$ are denoted as usual by $X_{i: \lambda}, 1 \leq i \leq \lambda$. Following David [4], the $Y$ variate associated with $X_{i: \lambda}$ is called the concomitant of the $i$ th order statistic and is denoted by $Y_{i: \lambda]}$. We consider the special case that the population density is

$$
f(x, y)=f(x \mid y) g(y),
$$

where $g(y)$ is the density of a probability distribution that is without loss of generality assumed to be standardized to have zero mean and unit variance, and where

$$
f(x \mid y)=\frac{1}{\sqrt{2 \pi} \vartheta} \exp \left(-\frac{1}{2}\left(\frac{x-y}{\vartheta}\right)^{2}\right)
$$

for a $\vartheta>0$. It is the purpose of this paper to obtain approximations for the expected values of the mean

$$
m_{1}=\frac{1}{\mu} \sum_{i=1}^{\mu} Y_{[\lambda-i+1: \lambda]}
$$

and the moments about the mean

$$
m_{j}=\frac{1}{\mu} \sum_{i=1}^{\mu}\left(Y_{[\lambda-i+1: \lambda]}-m_{1}\right)^{j}, \quad j \geq 2
$$


of the sample consisting of those $Y$-values that are associated with the $\mu$ largest $X$-values.

The determination of expected values of the moments in Eqs. (3) and (4) is a recurring problem in the theory of evolution strategies [3, 8]. Evolution strategies are powerful heuristics for numerical search and optimization. At time step $t$, the state of an evolution strategy includes a set of $\mu \geq 1$ candidate solutions to the problem at hand. A set of $\lambda>\mu$ new candidate solutions is generated from the existing set by means of certain operations that have the purpose of introducing variation. Subsequently, the $\mu$ best of the $\lambda$ candidate solutions thus generated are selected to replace the original set of candidate solutions in time step $t+1$. This form of selection is usually called truncation selection. Therefore, we refer to the set $\left\{Y_{\lambda-i+1: \lambda}: i=1, \ldots, \mu\right\}$ as the truncated sample even though this form of selection is commonly referred to as Type II censoring in Statistics. The quality of a candidate solution is of course determined by that candidate solution's objective function value. As real-world optimization problems almost always include sources of noise, it is of particular interest to consider the case that the observed or measured objective function values $X$ do not properly reflect the candidate solutions' true quality $Y_{i}$. The assumption of Gaussian noise is almost universal in the optimization literature and motivates the choice of probability density in Eq. (2). The quantity $\vartheta$ is referred to as the noise strength and determines the correlation coefficient

$$
a=\frac{1}{\sqrt{1+\vartheta^{2}}}
$$

of the bivariate distribution $F(x, y)$.

This paper is by no means the first to consider properties of concomitants of selected order statistics. In related work, Nagaraja [6] considered asymptotic properties of $m_{4}$ which he referred to as the induced selection differential. Yeo and David [9] developed a general expression for the probability that the $\mu$ objects that are selected include the $v \leq \mu$ objects with the largest $Y$-values. Nagaraja and David [7] derived limit distributions for the maximum $Y$-value of the truncated sample for both the extreme and the quantile cases. A survey of work concerned with all aspects of concomitants of order statistics has been compiled by David and Nagaraja [5].

The remainder of this paper is organized as follows. In Section 2, integral expressions for the expected values of sums of products of the concomitants of the selected order statistics are derived. In Section 3, an Edgeworth approximation is used for expressing the distribution of the $Y$ variates, making it possible to solve all but one of the integrals in the previously obtained expression. Even though only moments up to the fourth order are considered, there are no restrictions in principle that would prevent the inclusion of higher-order moments in the calculations. Then, a substitution is carried out with the goal of expressing the expected values of sums of products of the concomitants of the selected order statistics in terms of coefficients that can be obtained numerically. In the course of that substitution, a Cornish-Fisher expansion is used to express the inverse c.d.f. of the $X$ variates. Finally, in Section 4, expected values of the mean and of moments about the mean of the truncated sample are obtained. The special cases of the normal distribution - for which the results are exact and the $\chi^{2}$-distribution - for which it is shown experimentally that considering skewness and kurtosis in the calculations greatly improves the quality of the approximation, are discussed. Appendix A derives some identities that are used in Sections 2 and 3, and Appendix B contains a Mathematica program handling the tedious details of the calculations.

\section{Sums of products of concomitants}

In Section 4, we will express the moments of the truncated sample in terms of sums of products of the concomitants of the selected order statistics. Let $A=\left(\alpha_{1}, \ldots, \alpha_{v}\right)$ be a vector of $v \geq 1$ positive 
integers $\alpha_{j}, j=1, \ldots, v$, and let

$$
S_{A}=\sum Y_{\left[i_{1}: \lambda\right]}^{\alpha_{1}} \ldots Y_{\left[i_{v}: \lambda\right]}^{\alpha_{v}},
$$

where the summation ranges over all indices $i_{j}=\lambda-\mu+1, \lambda-\mu+2, \ldots, \lambda$ such that $i_{j} \neq i_{k}$ for any $j \neq k$. Note that for $v>\mu$, the summation is empty and $S_{A}=0$. So as to restrict the indices in $S_{A}$ such that $i_{1}<i_{2}<\cdots<i_{v}$, let us formally write $\pi_{A}\left(y_{1}, \ldots, y_{v}\right)$ for the sum of products of powers of the $y_{k}$ with all permutations of the exponents $\alpha_{1}, \ldots, \alpha_{v}$. For example,

$$
\begin{gathered}
\pi_{22}\left(y_{1}, y_{2}\right)=y_{1}^{2} y_{2}^{2} \\
\pi_{111}\left(y_{1}, y_{2}, y_{3}\right)=y_{1} y_{2} y_{3} \\
\pi_{211}\left(y_{1}, y_{2}, y_{3}\right)=y_{1}^{2} y_{2} y_{3}+y_{1} y_{2}^{2} y_{3}+y_{1} y_{2} y_{3}^{2} .
\end{gathered}
$$

Then we can write

$$
S_{A}=\sum_{i_{1}=\lambda-\mu+1}^{\lambda-v+1} \sum_{i_{2}=i_{1}+1}^{\lambda-v+2} \ldots \sum_{i_{v}=i_{v-1}+1}^{\lambda} \pi_{A}\left(Y_{\left[i_{1}: \lambda\right]}, \ldots, Y_{\left[i_{v}: \lambda\right]}\right) .
$$

The expected value of a sum of products of the concomitants of the selected order statistics with exponents prescribed by $A$ is thus

$$
\begin{aligned}
\mathrm{E}\left[S_{A}\right] & =\mathrm{E}\left[\sum_{i_{1}=\lambda-\mu+1}^{\lambda-v+1} \sum_{i_{2}=i_{1}+1}^{\lambda-v+2} \ldots \sum_{i_{v}=i_{v-1}+1}^{\lambda} \pi_{A}\left(Y_{\left[i_{1}: \lambda\right]}, \ldots, Y_{\left[i_{v}: \lambda\right]}\right)\right] \\
& =\int_{-\infty}^{\infty} \ldots \int_{-\infty}^{\infty} \sum_{i_{1}=\lambda-\mu+1}^{\lambda-v+1} \sum_{i_{2}=i_{1}+1}^{\lambda-v+2} \ldots \sum_{i_{v}=i_{v-1}+1}^{\lambda} \pi_{A}\left(y_{1}, \ldots, y_{v}\right) g_{\left[i_{1}, \ldots, i_{v}: \lambda\right]}\left(y_{1}, \ldots, y_{v}\right) \mathrm{d} y_{v} \ldots \mathrm{d} y_{1},
\end{aligned}
$$

where $g_{\left[i_{1}, \ldots, i_{v}: \lambda\right]}\left(y_{1}, \ldots, y_{v}\right)$ denotes the joint p.d.f. of the concomitants $Y_{\left[i_{k}: \lambda\right]}, k=1, \ldots, v$, with $1 \leq$ $i_{1}<i_{2}<\cdots<i_{v} \leq \lambda$. Using results quoted by Balakrishnan and Rao [2] and by David and Nagaraja [5], that joint p.d.f. can be written as

$$
\begin{aligned}
g_{\left[i_{1}, \ldots, i_{v}: \lambda\right]}\left(y_{1}, \ldots, y_{v}\right)=\lambda ! \int_{-\infty}^{\infty} \int_{x_{1}}^{\infty} \ldots \int_{x_{v-1}}^{\infty}\left[\prod_{k=1}^{v} g\left(y_{k}\right) f\left(x_{k} \mid y_{k}\right)\right] \\
{\left[\prod_{k=0}^{v} \frac{\left[F\left(x_{k+1}\right)-F\left(x_{k}\right)\right]^{i_{k+1}-i_{k}-1}}{\left(i_{k+1}-i_{k}-1\right) !}\right] \mathrm{d} x_{v} \ldots \mathrm{d} x_{2} \mathrm{~d} x_{1}, }
\end{aligned}
$$

where it is formally assumed that $i_{0}=0, i_{v+1}=\lambda+1, x_{0}=-\infty$, and $x_{v+1}=\infty$, and where $F(x)$ denotes the marginal c.d.f. of the $X$ variates. Therefore, exchanging the order of integrations and summations it follows that

$$
\begin{aligned}
\mathrm{E}\left[S_{A}\right]=\lambda ! & \int_{-\infty}^{\infty} \ldots \int_{-\infty}^{\infty} \pi_{A}\left(y_{1}, \ldots, y_{v}\right) \int_{-\infty}^{\infty} \int_{x_{1}}^{\infty} \ldots \int_{x_{v-1}}^{\infty}\left[\prod_{k=1}^{v} g\left(y_{k}\right) f\left(x_{k} \mid y_{k}\right)\right] \\
& \sum_{i_{1}=\lambda-\mu+1}^{\lambda-v+1} \sum_{i_{2}=i_{1}+1}^{\lambda-v+2} \ldots \sum_{i_{v}=i_{v-1}+1}^{\lambda}\left[\prod_{k=0}^{v} \frac{\left[F\left(x_{k+1}\right)-F\left(x_{k}\right)\right]^{i_{k+1}-i_{k}-1}}{\left(i_{k+1}-i_{k}-1\right) !}\right] \mathrm{d} x_{v} \ldots \mathrm{d} x_{2} \mathrm{~d} x_{1} \mathrm{~d} y_{v} \ldots \mathrm{d} y_{1} .
\end{aligned}
$$

Using Identity 1 from Appendix A it follows that

$$
\begin{array}{r}
\mathrm{E}\left[S_{A}\right]=\frac{\mu !(\lambda-\mu)}{(\mu-v) !}\left(\begin{array}{l}
\lambda \\
\mu
\end{array}\right) \int_{-\infty}^{\infty} \ldots \int_{-\infty}^{\infty} \pi_{A}\left(y_{1}, \ldots, y_{v}\right) \int_{-\infty}^{\infty} \int_{x_{1}}^{\infty} \ldots \int_{x_{v-1}}^{\infty}\left[\prod_{k=1}^{v} g\left(y_{k}\right) f\left(x_{k} \mid y_{k}\right)\right] \\
\int_{0}^{F\left(x_{1}\right)} z^{\lambda-\mu-1}[1-z]^{\mu-v} \mathrm{~d} z \mathrm{~d} x_{v} \ldots \mathrm{d} x_{2} \mathrm{~d} x_{1} \mathrm{~d} y_{v} \ldots \mathrm{d} y_{1} .
\end{array}
$$


Finally, letting $\Phi$ and $\phi$ denote the c.d.f and the p.d.f. of the standardized normal distribution, respectively, and substituting $z=\Phi(y)$ and exchanging the order of the integrations yields

$$
\mathrm{E}\left[S_{A}\right]=\frac{\mu !(\lambda-\mu)}{(\mu-v) !}\left(\begin{array}{l}
\lambda \\
\mu
\end{array}\right) \int_{-\infty}^{\infty} \phi(y)[\Phi(y)]^{\lambda-\mu-1}[1-\Phi(y)]^{\mu-v} I_{A}(y) \mathrm{d} y
$$

with

$$
\begin{aligned}
& I_{A}(y)=\int_{F^{-1}(\Phi(y))}^{\infty} \int_{x_{1}}^{\infty} \ldots \int_{x_{v-1}}^{\infty} \int_{-\infty}^{\infty} \ldots \int_{-\infty}^{\infty} \pi_{A}\left(y_{1}, \ldots, y_{v}\right) \\
& {\left[\prod_{k=1}^{v} g\left(y_{k}\right) f\left(x_{k} \mid y_{k}\right)\right] \mathrm{d} y_{v} \ldots \mathrm{d} y_{1} \mathrm{~d} x_{v} \ldots \mathrm{d} x_{2} \mathrm{~d} x_{1} . }
\end{aligned}
$$

That is, up to a single remaining integration, the problem of computing $\mathrm{E}\left[S_{A}\right]$ has been reduced to that of computing $I_{A}(y)$.

\section{Expanding the distributions}

So as to obtain a closed form expression for $I_{A}(y)$, let us expand the p.d.f. $g(y)$ in term of derivatives of the p.d.f. of the standard normal distribution. The first terms in the Edgeworth expansion of $g(y)$ read (Abramowitz and Stegun [1])

$$
g(y)=\phi(y)\left[1+\frac{\gamma_{1}}{6} \operatorname{He}_{3}(y)+\frac{\gamma_{2}}{24} \operatorname{He}_{4}(y)+\frac{\gamma_{1}^{2}}{72} \operatorname{He}_{6}(y)+\ldots\right],
$$

where $\gamma_{1}$ and $\gamma_{2}$ are the coefficients of skewness and kurtosis of the distribution, respectively, and where $\mathrm{He}_{k}(y)$ denotes the $k$ th Hermite polynomial. For the sake of brevity, we refrain from considering higher-order terms in the calculations. Note however that this is not a restriction in principle, and that additional terms could be considered.

Introducing new variables $x_{k}^{\prime}=x_{k} / \sqrt{1+\vartheta^{2}}, k=1, \ldots, v$, in Eq. (8) and using Eq. (2) yields

$$
I_{A}(y)={\sqrt{1+\vartheta^{2}}}^{v} \int_{x_{0}^{\prime}}^{\infty} \ldots \int_{x_{v-1}^{\prime}}^{\infty} J_{A}\left(x_{1}^{\prime}, \ldots, x_{v}^{\prime}\right) \mathrm{d} x_{v}^{\prime} \ldots \mathrm{d} x_{1}^{\prime},
$$

where $x_{0}^{\prime}=F^{-1}(\Phi(y)) / \sqrt{1+\vartheta^{2}}$, and where

$$
J_{A}\left(x_{1}^{\prime}, \ldots, x_{v}^{\prime}\right)=\frac{1}{\vartheta^{v}} \int_{-\infty}^{\infty} \ldots \int_{-\infty}^{\infty} \pi_{A}\left(y_{1}, \ldots, y_{v}\right)\left[\prod_{k=1}^{v} g\left(y_{k}\right) \phi\left(\frac{y_{k}-\sqrt{1+\vartheta^{2}} x_{k}^{\prime}}{\vartheta}\right)\right] \mathrm{d} y_{v} \ldots \mathrm{d} y_{1} .
$$

From Identity 2 in Appendix A with the correlation coefficient from Eq. (5) it follows that

$$
\frac{1}{\vartheta} \int_{-\infty}^{\infty} \operatorname{He}_{k}(y) \phi(y) \phi\left(\frac{y-\sqrt{1+\vartheta^{2}} x^{\prime}}{\vartheta}\right) \mathrm{d} y=a^{k+1} \operatorname{He}_{k}\left(x^{\prime}\right) \phi\left(x^{\prime}\right) .
$$

Therefore, using Eq. (9) and doing all $v$ integrations in Eq. (11), $J_{A}\left(x_{1}^{\prime}, \ldots, x_{v}^{\prime}\right)$ is of the form

$$
J_{A}\left(x_{1}^{\prime}, \ldots, x_{v}^{\prime}\right)=\left(\text { polynomial in }\left(x_{1}^{\prime}, \ldots, x_{v}^{\prime}\right)\right) \prod_{k=1}^{v} \phi\left(x_{k}^{\prime}\right),
$$


where the coefficients of the polynomial depend on $\gamma_{1}, \gamma_{2}$, and $a$ only. Using this result in Eq. (10), the remaining integrals can then be solved using Identities 3, 4, 5, and 6 from Appendix A. The final result is of the form

$$
I_{A}(y)=\sum_{i=0}^{v}\left(\text { polynomial in } x_{0}^{\prime}\right)_{i}\left[\phi\left(x_{0}^{\prime}\right)\right]^{\nu-i}\left[1-\Phi\left(x_{0}^{\prime}\right)\right]^{i},
$$

where again the coefficients of the polynomials depend on $\gamma_{1}, \gamma_{2}$, and $a$ only. The calculations are not difficult but tedious and lengthy. Written out, they occupy far more space than is available here. Therefore, instead of presenting detailed steps, we have included a Mathematica program in Appendix B that takes over the task of determining the coefficients of the polynomials in Eq. (14).

The result for $I_{A}(x)$ obtained thus far does not require any more integrations. However, it does depend on $x_{0}^{\prime}=F^{-1}(\Phi(y)) / \sqrt{1+\vartheta^{2}}$ rather than directly on $y$. The probability distribution of the $X$ variates is the convolution of the distribution of the $Y$ variates and a normal distribution with mean zero and with variance $\vartheta^{2}$. Therefore, its variance is $1+\vartheta^{2}$, its coefficient of skewness is $\gamma_{1} /{\sqrt{1+\vartheta^{2}}}^{3}=$ $a^{3} \gamma_{1}$, and its coefficient of kurtosis is $\gamma_{2} /\left(1+\vartheta^{2}\right)^{2}=a^{4} \gamma_{2}$. Expanding $F^{-1}(\Phi(y))$ into a CornishFisher series yields (Abramowitz and Stegun [1])

$$
\begin{aligned}
x_{0}^{\prime} & =\frac{F^{-1}(\Phi(y))}{\sqrt{1+\vartheta^{2}}} \\
& =y+\frac{\gamma_{1}}{6} a^{3} \operatorname{He}_{2}(y)+\frac{\gamma_{2}}{24} a^{4} \operatorname{He}_{3}(y)-\frac{\gamma_{1}^{2}}{36} a^{6}\left(2 \mathrm{He}_{3}(y)+\mathrm{He}_{1}(y)\right)+\ldots
\end{aligned}
$$

For notational brevity introducing

$$
d=\frac{\gamma_{1}}{6} a^{3}\left(y^{2}-1\right)+\frac{\gamma_{2}}{24} a^{4}\left(y^{3}-3 y\right)-\frac{\gamma_{1}^{2}}{36} a^{6}\left(2 y^{3}-5 y\right),
$$

it follows that $x_{0}^{\prime}=y+d+\ldots$ and therefore by Taylor expansion around $y$ that

$$
1-\Phi\left(x_{0}^{\prime}\right)=1-\Phi(y)-\phi(y)\left(d-\frac{y d^{2}}{2}+\ldots\right)
$$

and that

$$
\phi\left(x_{0}^{\prime}\right)=\phi(y)\left(1-y d+\frac{\left(y^{2}-1\right) d^{2}}{2}+\ldots\right) .
$$

Binomially expanding powers of these quantities it follows that

$$
x_{0}^{\prime k}=y^{k}+k y^{k-1} d+\frac{k(k-1)}{2} y^{k-2} d^{2}+\ldots,
$$

that

$$
\begin{aligned}
{\left[1-\Phi\left(x_{0}^{\prime}\right)\right]^{k}=[1-\Phi(y)]^{k}-k[1-\Phi(y)]^{k-1} } & \phi(y)\left(d-\frac{y d^{2}}{2}+\ldots\right) \\
& +\frac{k(k-1)}{2}[1-\Phi(y)]^{k-2}[\phi(y)]^{2}\left(d^{2}+\ldots\right)+\ldots,
\end{aligned}
$$

and that

$$
\left[\phi\left(x_{0}^{\prime}\right)\right]^{k}=[\phi(y)]^{k}\left[1-k\left(y d-\frac{\left(y^{2}-1\right) d^{2}}{2}+\ldots\right)+\frac{k(k-1)}{2}\left(y^{2} d^{2}+\ldots\right)+\ldots\right] .
$$


Note that $d^{2}=\gamma_{1}^{2} a^{6}\left(y^{2}-1\right)^{2} / 36+\ldots$, and that all terms represented by dots consists of higher-order terms only. Inserting Eqs. (15), (16), and (17) in Eq. (14) results in

$$
I_{A}(y)=\sum_{i=0}^{v}\left[Z_{i, 0}^{(A)}(y)+\frac{\gamma_{1}}{6} Z_{i, 1}^{(A)}(y)+\frac{\gamma_{2}}{24} Z_{i, 2}^{(A)}(y)+\frac{\gamma_{1}^{2}}{36} Z_{i, 3}^{(A)}(y)+\ldots\right][\phi(y)]^{v-i}[1-\Phi(y)]^{i},
$$

where the

$$
Z_{i, j}^{(A)}(y)=\sum_{k \geq 0} \zeta_{i, j}^{(A)}(k) \operatorname{He}_{k}(y)
$$

are polynomials in $y$. The coefficients $\zeta_{i, j}^{(A)}(k)$ that have been obtained by straightforward but lengthy calculations and that can alternatively be arrived at using the Mathematica program in Appendix B are listed in Table 1.

Finally, inserting Eq. (18) in Eq. (7) yields

$$
\begin{array}{r}
\mathrm{E}\left[S_{A}\right]=\frac{\mu !(\lambda-\mu)}{(\mu-v) !}\left(\begin{array}{l}
\lambda \\
\mu
\end{array}\right) \sum_{i=0}^{v} \int_{-\infty}^{\infty}\left[Z_{i, 0}^{(A)}(y)+\frac{\gamma_{1}}{6} Z_{i, 1}^{(A)}(y)+\right. \\
\left.\frac{\gamma_{2}}{24} Z_{i, 2}^{(A)}(y)+\frac{\gamma_{1}^{2}}{36} Z_{i, 3}^{(A)}(y)+\ldots\right] \\
{[\phi(y)]^{v-i+1}[\Phi(y)]^{\lambda-\mu-1}[1-\Phi(y)]^{\mu-v+i} \mathrm{~d} y .}
\end{array}
$$

The remaining integration usually cannot be done analytically. Instead, we choose to define coefficients

$$
h_{\mu, \lambda}^{i, k}=(\lambda-\mu)\left(\begin{array}{l}
\lambda \\
\mu
\end{array}\right) \int_{-\infty}^{\infty} \operatorname{He}_{k}(y)[\phi(y)]^{i+1}[\Phi(y)]^{\lambda-\mu-1}[1-\Phi(y)]^{\mu-i} \mathrm{~d} y
$$

that can be computed numerically without difficulties. With these coefficients it follows

$$
\mathrm{E}\left[S_{A}\right]=\frac{\mu !}{(\mu-v) !} \sum_{i=0}^{v} \sum_{k \geq 0}\left[\zeta_{i, 0}^{(A)}(k)+\frac{\gamma_{1}}{6} \zeta_{i, 1}^{(A)}(k)+\frac{\gamma_{2}}{24} \zeta_{i, 2}^{(A)}(k)+\frac{\gamma_{1}^{2}}{36} \zeta_{i, 3}^{(A)}(k)+\ldots\right] h_{\mu, \lambda}^{\nu-i, k}
$$

for the expected value of the sum of products of the concomitants of the selected order statistics with exponents prescribed by $A$.

\section{Expected values of moments of the truncated sample}

The mean and the moments about the mean of the truncated sample can be expressed in terms of the $S_{A}$ simply by multiplying out Eq. (4) and rearranging terms. It is easily verified that

$$
\begin{gathered}
m_{1}=\frac{1}{\mu} S_{1} \\
m_{2}=\frac{\mu-1}{\mu^{2}} S_{2}-\frac{2}{\mu^{2}} S_{11} \\
m_{3}=\frac{(\mu-1)(\mu-2)}{\mu^{3}} S_{3}-3 \frac{\mu-2}{\mu^{3}} S_{21}+\frac{12}{\mu^{3}} S_{111} \\
m_{4}=\frac{(\mu-1)\left(\mu^{2}-3 \mu+3\right)}{\mu^{4}} S_{4}-4 \frac{\mu^{2}-3 \mu+3}{\mu^{4}} S_{31}+6 \frac{2 \mu-3}{\mu^{4}} S_{22}+12 \frac{\mu-3}{\mu^{4}} S_{211}-\frac{72}{\mu^{4}} S_{1111} .
\end{gathered}
$$




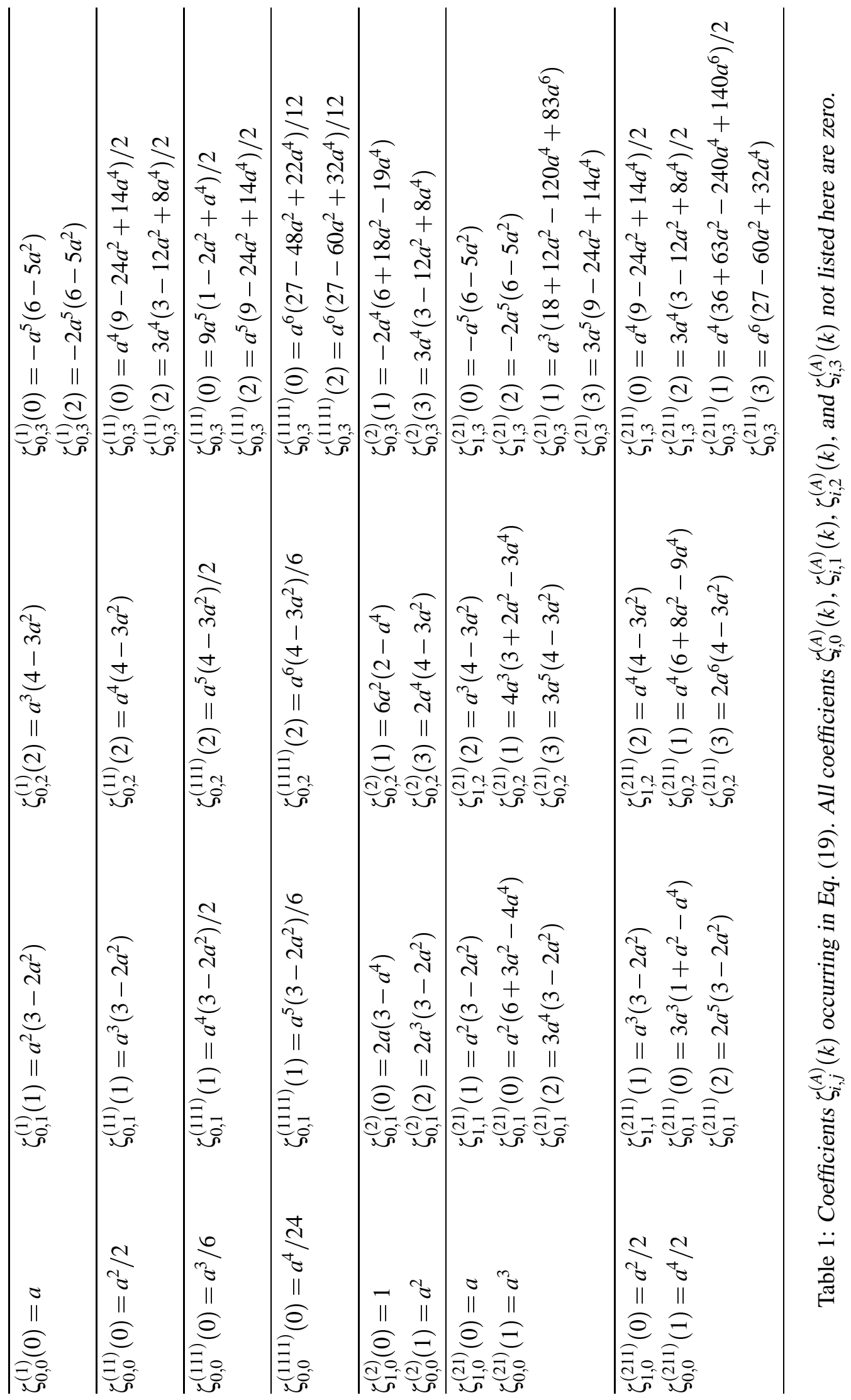




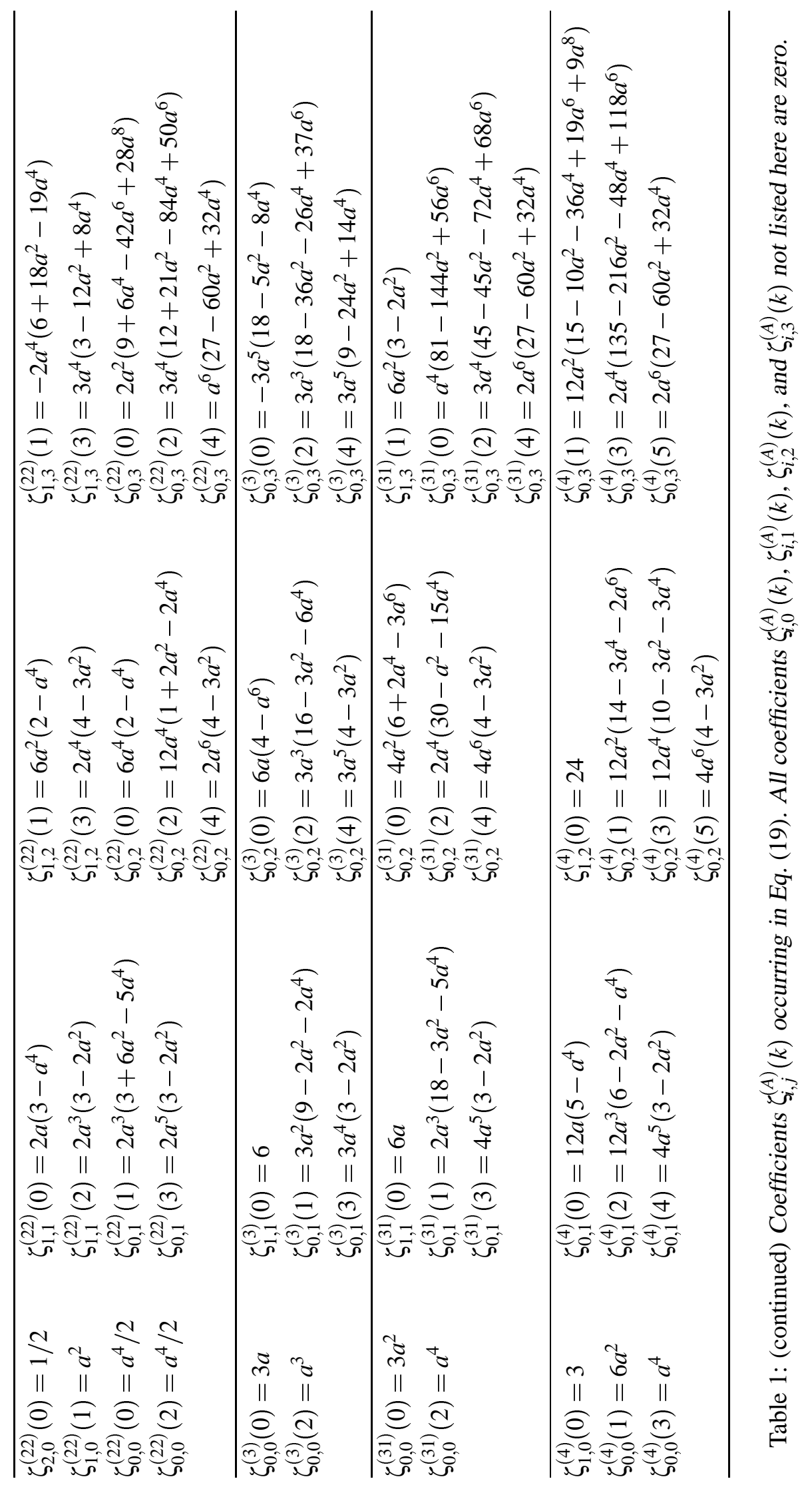


In some situations, it is also useful to know the expected value of the square of the variance of the truncated sample. Squaring Eq. (4) and multiplying out, it follows that

$$
m_{2}^{2}=\frac{(\mu-1)^{2}}{\mu^{4}} S_{4}-4 \frac{\mu-1}{\mu^{4}} S_{31}+2 \frac{\mu^{2}-2 \mu+3}{\mu^{4}} S_{22}-4 \frac{\mu-3}{\mu^{4}} S_{211}+\frac{24}{\mu^{4}} S_{1111} .
$$

Thus, using Equation (21) and the coefficients from Table 1, expected values of the mean and of the moments about the mean of the truncated sample can easily be determined. For the special case that the distribution of the $Y$ variate is normal, the results read

$$
\begin{gathered}
\mathrm{E}\left[m_{1}\right]=a h_{\mu, \lambda}^{1,0} \\
\mathrm{E}\left[m_{2}\right]=\frac{\mu-1}{\mu}\left[1-a^{2}\left(h_{\mu, \lambda}^{2,0}-h_{\mu, \lambda}^{1,1}\right)\right] \\
\mathrm{E}\left[m_{3}\right]=\frac{(\mu-1)(\mu-2)}{\mu^{2}} a^{3}\left[h_{\mu, \lambda}^{1,2}-3 h_{\mu, \lambda}^{2,1}+2 h_{\mu, \lambda}^{3,0}\right] \\
\mathrm{E}\left[m_{4}\right]=\frac{(\mu-1)\left(\mu^{2}-3 \mu+3\right)}{\mu^{3}}\left[6-12 a^{2}\left(h_{\mu, \lambda}^{2,0}-h_{\mu, \lambda}^{1,1}\right)+a^{4}\left(h_{\mu, \lambda}^{1,3}-h_{\mu, \lambda}^{2,2}+3 h_{\mu, \lambda}^{2,0}\right)\right] \\
-3 \frac{(\mu-1)(\mu-2)(\mu-3)}{\mu^{3}}\left[1-2 a^{2}\left(h_{\mu, \lambda}^{2,0}-h_{\mu, \lambda}^{1,1}\right)+a^{4}\left(h_{\mu, \lambda}^{2,0}+h_{\mu, \lambda}^{2,2}-2 h_{\mu, \lambda}^{3,1}+h_{\mu, \lambda}^{4,0}\right)\right] \\
\mathrm{E}\left[m_{2}^{2}\right]=\frac{(\mu-1)^{2}}{\mu^{3}}\left[6-12 a^{2}\left(h_{\mu, \lambda}^{2,0}-h_{\mu, \lambda}^{1,1}\right)+a^{4}\left(h_{\mu, \lambda}^{1,3}-h_{\mu, \lambda}^{2,2}+3 h_{\mu, \lambda}^{2,0}\right)\right] \\
+\frac{(\mu-1)(\mu-2)(\mu-3)}{\mu^{3}}\left[1-2 a^{2}\left(h_{\mu, \lambda}^{2,0}-h_{\mu, \lambda}^{1,1}\right)+a^{4}\left(h_{\mu, \lambda}^{2,0}+h_{\mu, \lambda}^{2,2}-2 h_{\mu, \lambda}^{3,1}+h_{\mu, \lambda}^{4,0}\right)\right] .
\end{gathered}
$$

Note that those results are in fact exact for the case of a normally distributed population.

If the population is not normal, the approach pursued is merely an approximation. As the resulting expressions taking skewness and kurtosis of the population into account are rather long, we refrain from writing them down explicitly. However, they can be obtained easily from Table 1. So as to verify that the approach yields good results for distributions other than the normal, we considered the case of a standardized $\chi_{n}^{2}$-distribution. This choice of distribution to consider is motivated by problems in the theory of evolution strategies, where optimization in high-dimensional search spaces requires considering variables that are sums of independent components that can in a first approximation be assumed to have normal distribution. As the $j$ th cumulant of the standardized $\chi_{n}^{2}$-distribution is of order $n^{1-j / 2}$ with respect to $n$, the error in the approximation of the expected values of the moments of the truncated sample is of order $O\left(n^{-1 / 2}\right)$ if the expansions in Section 3 are cut off after the first term, it is of order $O\left(n^{-1}\right)$ if the coefficient of skewness $\gamma_{1}$ is included, and it is of order $O\left(n^{-3 / 2}\right)$ if moments up to the fourth order are considered. The results for $n=10$ and for $\mu=3$ and $\lambda=10$ are illustrated in Fig. 1. In that case, the coefficient of skewness is $\gamma_{1}=\sqrt{0.8}$, the coefficient of kurtosis is $\gamma_{2}=1.2$. The value of $n=10$ is rather small, and more exact results are achieved for greater $n$. Nonetheless, it can be seen that including the skewness and the kurtosis of the population distribution in the calculations substantially improves the quality of the results, and that especially the values computed for the first and second moments very closely reflect the measured values. 

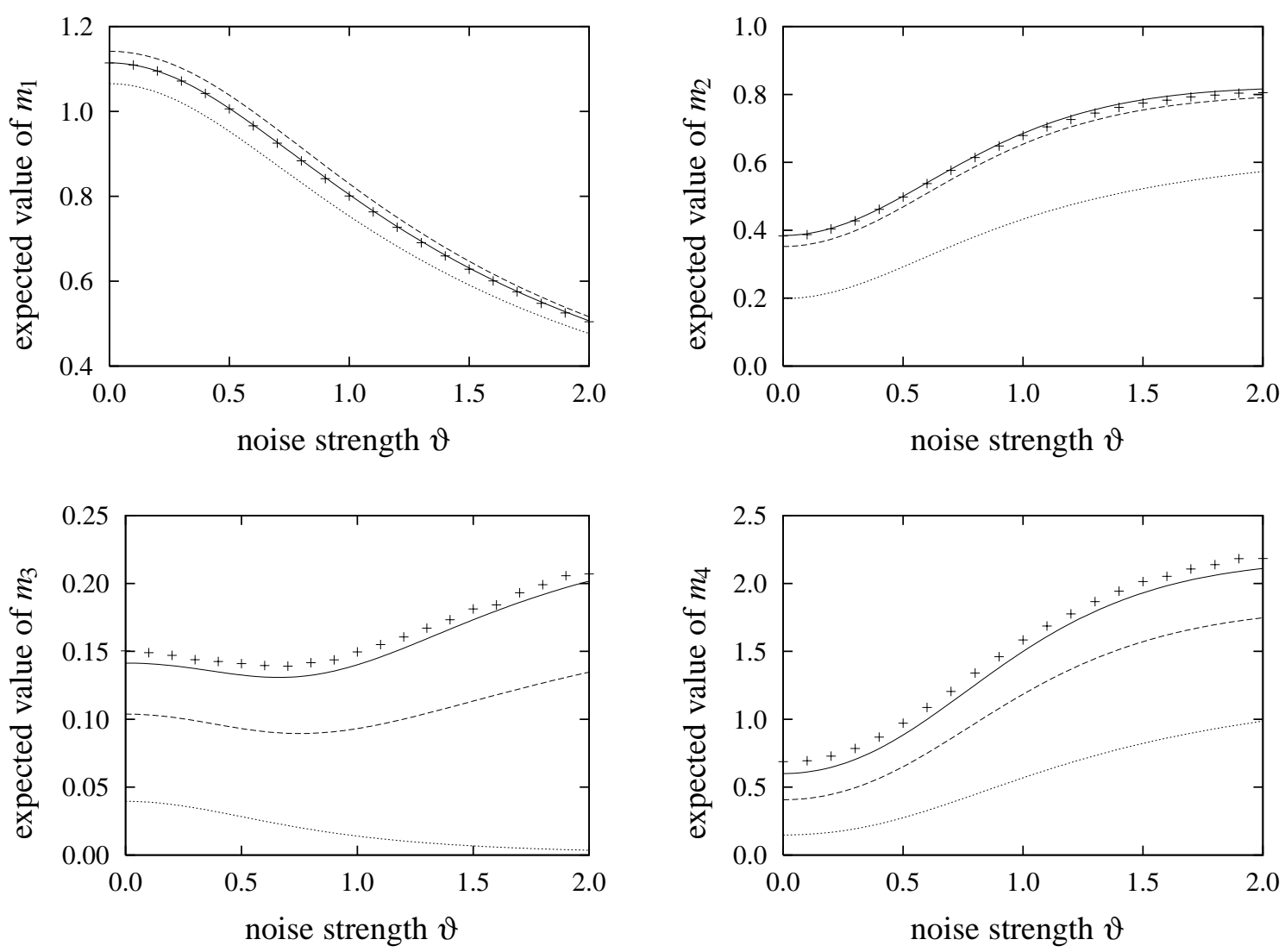

Figure 1: Expected values of the mean $m_{1}$ and of the moments about the mean $m_{2}, m_{3}$, and $m_{4}$ of truncated samples as functions of the noise strength $\vartheta$. The distribution from which the $\lambda=10$ sample members are drawn is a standardized $\chi_{10}^{2}$-distribution, the size of the truncated sample is $\mu=3$. The crosses represent measurements from computer experiments, the lines have been obtained using the approximation from Section 4. The solid lines result from considering the coefficients of skewness as well as kurtosis, the dashed lines from considering the coefficient of skewness only, and the dotted lines represent the case that only the variance of the distribution is taken into account.

\section{A Some useful identities}

Identity 1: For integer $v>0$ and for any integers $\mu$ and $\lambda$ with $v \leq \mu<\lambda$ and real numbers $F_{k}$, $k=1,2, \ldots, v$, the identity

$$
\begin{aligned}
\sum_{i_{1}=\lambda-\mu+1}^{\lambda-v+1} \sum_{i_{2}=i_{1}+1}^{\lambda-v+2} \cdots \sum_{i_{v}=i_{v-1}+1}^{\lambda} \prod_{k=0}^{v} \frac{\left[F_{k+1}-F_{k}\right]^{i_{k+1}-i_{k}-1}}{\left(i_{k+1}-i_{k}-1\right) !} & \\
= & \frac{1}{(\lambda-\mu-1) !(\mu-v) !} \int_{0}^{F_{1}} z^{\lambda-\mu-1}[1-z]^{\mu-v} \mathrm{~d} z
\end{aligned}
$$

where formally $F_{0}=0, F_{\mathrm{v}+1}=1, i_{0}=0$, and $i_{v+1}=\lambda+1$ are assumed, holds.

Identity 2: For non-negative integer $k$ and real numbers $\mu$ and $\sigma$, the identity

$$
\int_{-\infty}^{\infty} \operatorname{He}_{k}(x) \phi(x) \phi\left(\frac{x-\mu}{\sigma}\right) \mathrm{d} x=\frac{\sigma}{\left(1+\sigma^{2}\right)^{(k+1) / 2}} \mathrm{He}_{k}\left(\frac{\mu}{\left(1+\sigma^{2}\right)^{1 / 2}}\right) \phi\left(\frac{\mu}{\left(1+\sigma^{2}\right)^{1 / 2}}\right)
$$


holds.

Identity 3: For non-negative integer $k$ and real number $z$, the identity

$$
\int_{z}^{\infty} \mathrm{He}_{k}(x) \phi(x) \mathrm{d} x= \begin{cases}1-\Phi(z) & \text { if } k=0 \\ \operatorname{He}_{k-1}(z) \phi(z) & \text { if } k>0\end{cases}
$$

holds.

Identity 4: For positive integer $k$ and real numbers $z$ and $\beta \neq 0$, the identity

$$
\begin{aligned}
\int_{z}^{\infty} \operatorname{He}_{k}(x)[\phi(x)]^{\beta} \mathrm{d} x & \\
& = \begin{cases}\frac{1}{\beta}[\phi(z)]^{\beta} & \text { if } k=1 \\
\frac{1}{\beta} \mathrm{He}_{k-1}(z)[\phi(z)]^{\beta}-\frac{\beta-1}{\beta}(k-1) \int_{z}^{\infty} \mathrm{He}_{k-2}(x)[\phi(x)]^{\beta} \mathrm{d} x & \text { if } k>1\end{cases}
\end{aligned}
$$

holds.

Identity 5: For non-negative integer $k$ and real number $z$, the identity

$$
\begin{aligned}
& \int_{z}^{\infty} \mathrm{He}_{k}(x) \phi(x)[1-\Phi(x)] \mathrm{d} x \\
&= \begin{cases}\frac{1}{2}[1-\Phi(x)]^{2} & \text { if } k=0 \\
\mathrm{He}_{k-1}(z) \phi(z)[1-\Phi(z)]-\int_{z}^{\infty} \mathrm{He}_{k-1}(x)[\phi(x)]^{2} \mathrm{~d} x & \text { if } k>0\end{cases}
\end{aligned}
$$

holds.

Identity 6: For positive integer $k$ and real number $z$, the identity

$$
\begin{aligned}
& \int_{z}^{\infty} \operatorname{He}_{k}(x)[\phi(x)]^{2} {[1-\Phi(x)] \mathrm{d} x } \\
&=\left\{\begin{array}{rr}
\frac{1}{2}[\phi(z)]^{2}[1-\Phi(z)]-\frac{1}{2} \int_{z}^{\infty}[\phi(x)]^{3} \mathrm{~d} x & \text { if } k=1 \\
\frac{1}{2} \mathrm{He}_{k-1}(z)[\phi(z)]^{2}[1-\Phi(z)]-\frac{1}{2} \int_{z}^{\infty} \operatorname{He}_{k-1}(x)[\phi(x)]^{3} \mathrm{~d} x & \text { if } k>1 \\
-\frac{k-1}{2} \int_{z}^{\infty} \operatorname{He}_{k-2}(x)[\phi(x)]^{2}[1-\Phi(x)] \mathrm{d} x &
\end{array}\right.
\end{aligned}
$$

holds.

Proofs: All identities are shown by induction. The proof of Identity 2 relies on the use of quadratic completion of the argument of the exponential function. The proofs of Identities 3-6 are straightforward if integration by parts and the properties

$$
\frac{\mathrm{d}}{\mathrm{d} x} \mathrm{He}_{k}(x)=k \mathrm{He}_{k-1}(x)
$$


and

$$
\operatorname{He}_{k+1}(x)=x \mathrm{He}_{k}(x)-k \mathrm{He}_{k-1}(x)
$$

of Hermite polynomials are used.

The proof of Identity 1 is a bit more involved. Let us write $l h s_{\mu, \lambda}(v)$ and $r h s_{\mu, \lambda}(v)$ for the left and right hand sides of Identity 1 , respectively, and let $\mu<\lambda$. Then,

$$
\begin{aligned}
\operatorname{lhs}_{\mu, \lambda}(1) & =\sum_{i=\lambda-\mu+1}^{\lambda} \frac{\left[1-F_{1}\right]^{\lambda-i} F_{1}^{i-1}}{(\lambda-i) !(i-1) !} \\
& =\sum_{j=1}^{\mu} \frac{F_{1}^{\lambda-j}\left[1-F_{1}\right]^{j-1}}{(\lambda-j) !(j-1) !} .
\end{aligned}
$$

According to Eqs. 6.6.4 and 26.5.1 in [1] it follows in terms of the incomplete regularized Beta function that

$$
\begin{aligned}
\operatorname{lhs}_{\mu, \lambda}(1) & =\frac{1}{(\lambda-1) !} I_{F_{1}}(\lambda-\mu, \mu) \\
& =\frac{1}{(\lambda-\mu-1) !(\mu-1) !} \int_{0}^{F_{1}} z^{\lambda-\mu-1}[1-z]^{\mu-1} \mathrm{~d} z \\
& =r h s_{\mu, \lambda}(1),
\end{aligned}
$$

and the validity of the identity for $\mathrm{v}=1$ has been shown.

For the inductive step, let us now assume that the identity holds for a particular value of $v$ and all values of $\mu$ and $\lambda$ that satisfy $v \leq \mu<\lambda$. To show that the identity holds for $\nu+1$, let $\mu$ and $\lambda$ satisfy $v+1 \leq \mu<\lambda$. The left hand side of the identity then reads

$$
\operatorname{lh} s_{\mu, \lambda}(v+1)=\sum_{i_{1}=\lambda-\mu+1}^{\lambda-v} \sum_{i_{2}=i_{1}+1}^{\lambda-v+1} \ldots \sum_{i_{v+1}=i_{v}+1}^{\lambda} \prod_{k=0}^{v+1} \frac{\left[F_{k+1}-F_{k}\right]^{i_{k+1}-i_{k}-1}}{\left(i_{k+1}-i_{k}-1\right) !},
$$

where $F_{0}=0, F_{v+2}=1, i_{0}=0$, and $i_{v+2}=\lambda+1$. The innermost sum is

$$
\begin{aligned}
\sum_{i_{v+1}=i_{v}+1}^{\lambda} \prod_{k=0}^{v+1} \frac{\left[F_{k+1}-F_{k}\right]^{i_{k+1}-i_{k}-1}}{\left(i_{k+1}-i_{k}-1\right) !} \\
=\left[\prod_{k=0}^{v-1} \frac{\left[F_{k+1}-F_{k}\right]^{i_{k+1}-i_{k}-1}}{\left(i_{k+1}-i_{k}-1\right) !}\right] \sum_{i_{v+1}=i_{v}+1}^{\lambda} \frac{\left[1-F_{v+1}\right]^{\lambda-i_{v+1}}\left[F_{v+1}-F_{v}\right]^{i_{v+1}-i_{v}-1}}{\left(\lambda-i_{v+1}\right) !\left(i_{v+1}-i_{v}-1\right) !} \\
=\left[\prod_{k=0}^{v-1} \frac{\left[F_{k+1}-F_{k}\right]^{i_{k+1}-i_{k}-1}}{\left(i_{k+1}-i_{k}-1\right) !}\right] \sum_{j=0}^{\lambda-i_{v}-1} \frac{\left[1-F_{v+1}\right]^{\lambda-i_{v}-1-j}\left[F_{v+1}-F_{v}\right]^{j}}{\left(\lambda-i_{v}-1-j\right) ! j !} \\
=\left[\prod_{k=0}^{v-1} \frac{\left[F_{k+1}-F_{k}\right]^{i_{k+1}-i_{k}-1}}{\left(i_{k+1}-i_{k}-1\right) !}\right] \frac{\left[1-F_{v}\right]^{\lambda-i_{v}-1}}{\left(\lambda-i_{v}-1\right) !} \\
=\prod_{k=0}^{v} \frac{\left[F_{k+1}-F_{k}\right]_{k+1}^{i_{k+1}-i_{k}-1}}{\left(i_{k+1}-i_{k}-1\right) !},
\end{aligned}
$$

where the third step follows from the binomial theorem and where in the last line $F_{v+1}=1$ and $i_{v+1}=\lambda$. It thus follows that

$$
\begin{aligned}
l h s_{\mu, \lambda}(v+1) & =\sum_{i_{1}=\lambda-\mu+1}^{\lambda-v} \sum_{i_{2}=i_{1}+1}^{\lambda-v+1} \cdots \sum_{i_{v}=i_{v-1}+1}^{\lambda-1} \prod_{k=0}^{v} \frac{\left[F_{k+1}-F_{k}\right]^{i_{k+1}-i_{k}-1}}{\left(i_{k+1}-i_{k}-1\right) !} \\
& =\operatorname{lhs}_{\mu-1, \lambda-1}(v) .
\end{aligned}
$$


As $v \leq \mu-1<\lambda-1$ and as the identity holds for $v$ it follows that

$$
\begin{aligned}
l h s_{\mu, \lambda}(v+1) & =r h s_{\mu-1, \lambda-1}(v) \\
& =\frac{1}{(\lambda-\mu-1) !(\mu-v) !} \int_{0}^{F_{1}} z^{\lambda-\mu-1}[1-z]^{\mu-v} \mathrm{~d} z \\
& =r h s_{\mu, \lambda}(v+1)
\end{aligned}
$$

and the validity of the identity has been shown.

\section{B Mathematica code}

This section contains the Mathematica code used to compute the coefficients in Table 1. Let us first define Hermite polynomials:

Hermite [k_, x_] := Simplify [HermiteH [k, x/Sqrt [2] ]/Sqrt [2] ^k] ;

This is necessary as Mathematica's built-in definition HermiteH differs from our definition. So as to compose the integrand in Eq. (11) we define

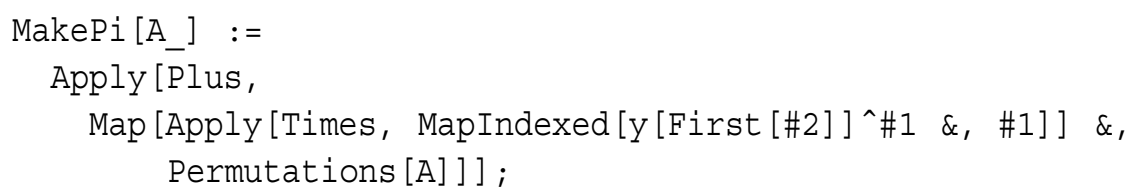

where A stands for the exponent vector $A$, to compute $\pi_{A}\left(y_{1}, \ldots, y_{v}\right)$ and subsequently

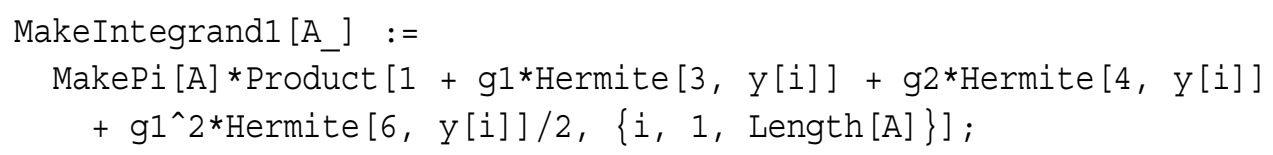

to obtain the integrand. Here, $g 1$ and g2 stand for $\gamma_{1} / 6$ and $\gamma_{2} / 24$, respectively. Note that for simplicity we have omitted the terms involving exponential functions from the integrand and that therefore we will need to give integration rules that take that into account.

Before implementing the proper integration routines, let us define a useful routine for expanding polynomials in terms of their Hermite basis:

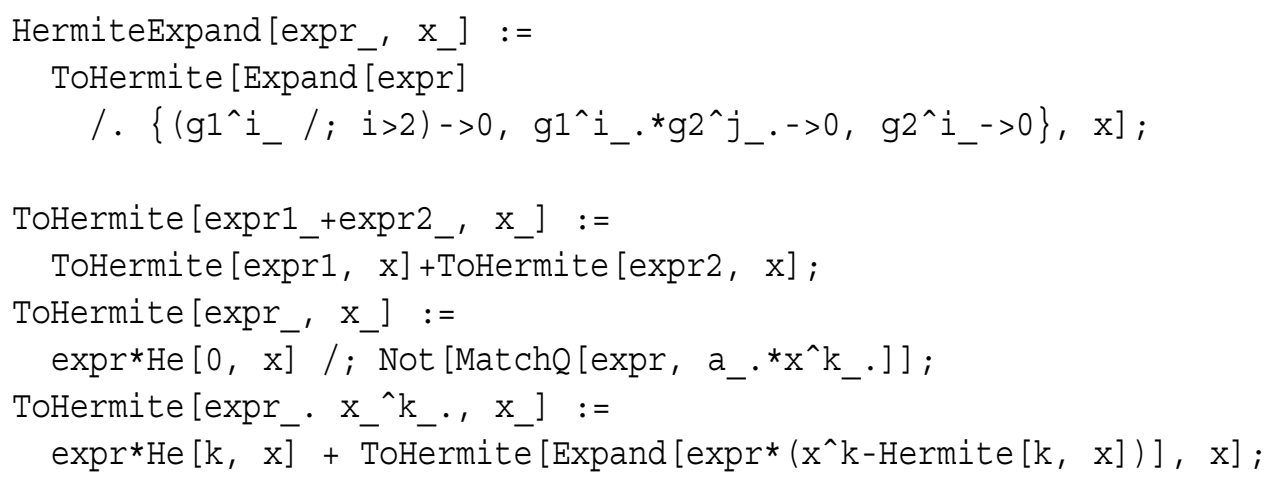

HermiteExpand both expresses powers of the $y[i]$ as linear combinations of Hermite polynomials and it eliminates terms involving higher-order cumulants. Note that we use He rather than Hermite as we do not want Mathematica to reverse the effect of applying ToHermite. Writing x [i] and y [i] for the $x_{i}^{\prime}$ and the $y_{i}$, respectively, the $v$-fold integration leading to the representation given in Eq. (13) is then handled by 


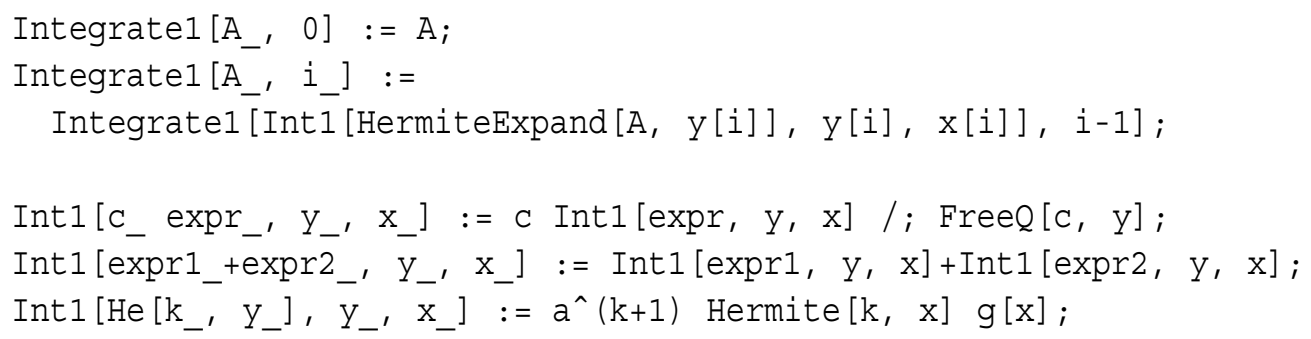

where Int1 implements the integration rule Eq. (12) taking into account that the terms involving exponential functions had been left out, and where the second argument of Integratel needs to be $v$ initially. Here and in what follows, $\mathrm{f}[\mathrm{y}]$ and $\mathrm{g}[\mathrm{y}]$ stand for $1-\Phi(y)$ and $\phi(y)$, respectively. The integrand in Eq. (10) is then simply given by:

MakeIntegrand2 [A_] := Integrate1 [MakeIntegrand1 $[\mathrm{A}]$, Length $[\mathrm{A}]]$;

where again A stands for the exponent vector $A$. The $v$-fold integration in Eq. (10) is done by

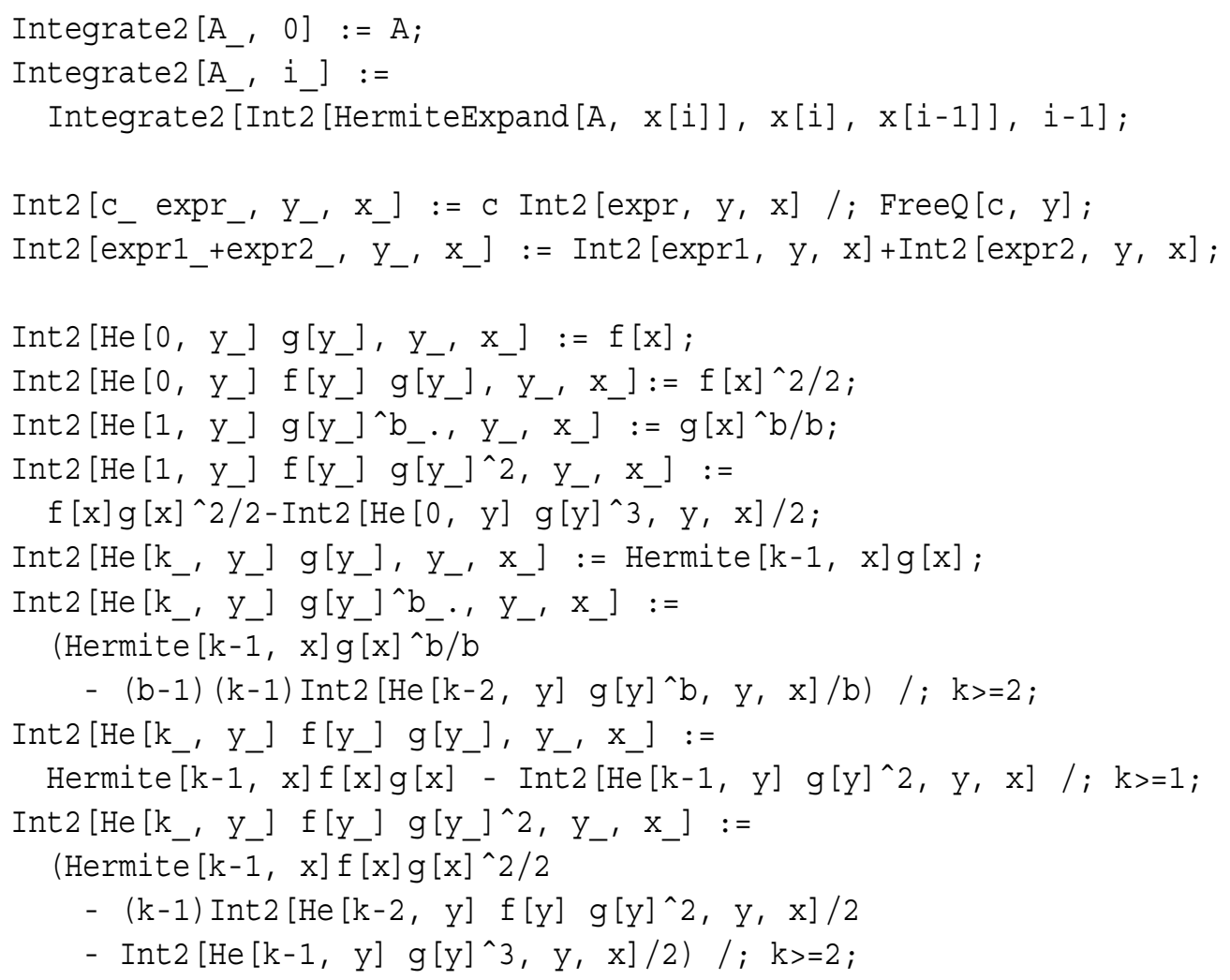

where Int2 implements the integration rules given by Identities 3, 4, 5, and 6 from Appendix A and where the second argument of Integrate 2 needs to be $v$ initially.

The result of the steps so far is the representation of $I_{A}(y)$ given by Eq. (14). To do the substitution prescribed by Eqs (15), (16), and (17) we define:

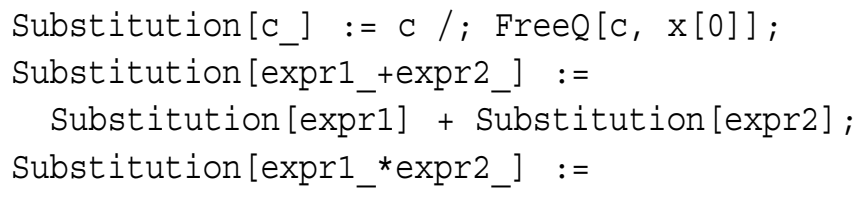




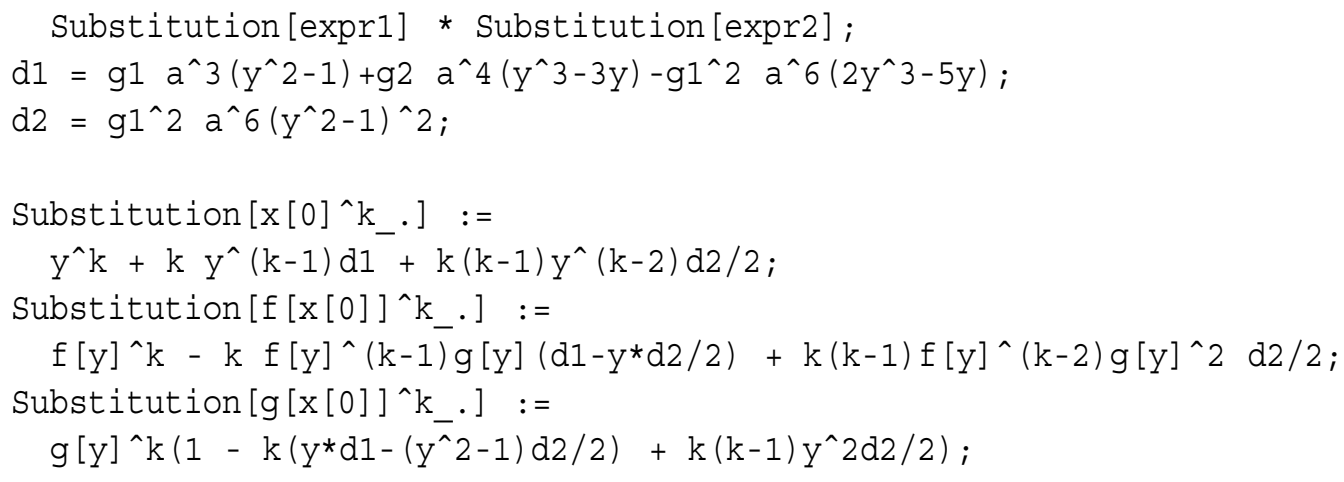

Finally, the representation of $I_{A}(y)$ given by Eq. (18) can be obtained by:

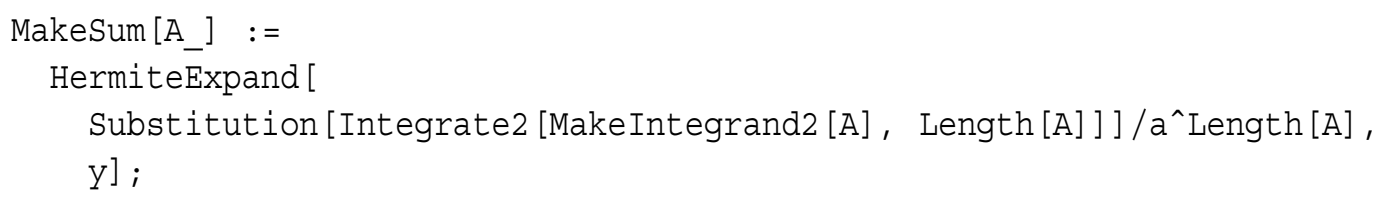

where the division by $a^{v}$ reflects the factors $\sqrt{1+\vartheta^{2}}$ in Eq. (10).

So as to print the coefficients in table form appropriate for entering them in Table 1, let us define:

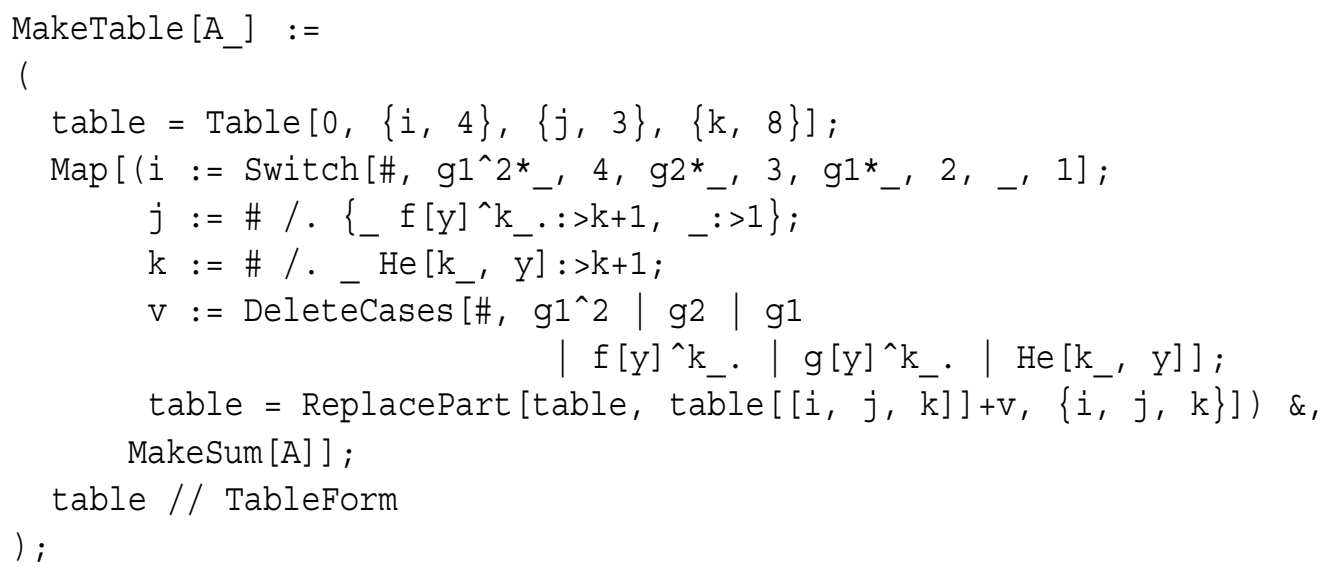

Calling for example MakeTable $[\{2,1,1\}]$ then arranges the coefficients in a table with $\zeta_{i, j}^{211}(k)$ in row $k$ and column $j$.

\section{Acknowledgments}

This work was supported by the Deutsche Forschungsgemeinschaft (DFG) under grants Be1578/4-2 and Be1578/6-3. The publication of the work was also supported by the DFG as part of the Collaborative Research Center "Computational Intelligence" (SFB 531). Hans-Georg Beyer is a Heisenberg Fellow of the DFG.

\section{References}

[1] Abramowitz, M. And Stegun, I. A. (1965). Handbook of Mathematical Functions. Dover, New York. 
[2] Balakrishnan, N. And RaO, C. R. (1998). Order statistics: An introduction. In Handbook of Statistics 16, eds. N. Balakrishnan and C. R. Rao. Elsevier, Amsterdam, pp. 3-24.

[3] Beyer, H.-G. (2001). The Theory of Evolution Strategies. Springer, Berlin.

[4] David, H. A. (1973). Concomitants of order statistics. Bull. Internat. Statist. Inst. 45, 295300.

[5] David, H. A. And Nagaraja, H. N. (1998). Concomitants of order statistics. In Handbook of Statistics 16, eds. N. Balakrishnan and C. R. Rao. Elsevier, Amsterdam, pp. 487-513.

[6] Nagaraja, H. N. (1982). Some asymptotic results for the induced selection differential. J. Appl. Prob. 19, 253-261.

[7] Nagaraja, H. N. AND David, H. A. (1994). Distribution of the maximum of concomitants of selected order statistics. Ann. Statist. 22, 478-494.

[8] Rudolph, G. (1997). Convergence Properties of Evolutionary Algorithms. Dr. Kovač, Hamburg.

[9] YAO, W. B. AND DAVID, H. A. (1984). Selection through an associated characteristic, with applications to the random effects model. J. Amer. Statist. Soc. 79, 399-405. 